\title{
Long-wavelength spectral studies of giant radio sources
}

\author{
Emanuela Orrù $\grave{1}^{1,2}$ \\ ${ }^{1}$ Dipartimento di Fisica, Università degli Studi di Cagliari, \\ Cittadella Universitaria, I-09042 Monserrato (CA), Italy \\ email: eorru_s@ira.inaf.it \\ ${ }^{2}$ INAF - Osservatorio Astronomico di Cagliari, \\ Loc. Poggio dei Pini, Strada 54, I-09012 Capoterra (CA), Italy
}

Abstract. We study giant radio sources at long wavelengths.

Keywords. giant radio sources

We have performed a long-wavelength spectral study of 3C 35 and 3C 223. These are two giant radio sources selected from a sub-sample of giant radio sources taken from the Lara et al. (2001) and Ishwara-Chandra (1999) samples.

Giant radio sources (GRS) are defined as those objects with a projected linear size larger than $1 \mathrm{Mpc}\left(H_{0}=50 \mathrm{~km} \mathrm{~s}^{-1} \mathrm{Mpc}^{-1}, q_{0}=0.5\right)$.

We produced new Very Large Array images at 74 and $327 \mathrm{MHz}$. Spectral analyses have been made, including $1.4 \mathrm{GHz}$ images from the literature. For both sources, in this range of frequencies, the spectral shape follows a power law in the hot spot with a slope of $\alpha \simeq 0.5-0.6$. In the inner region of the lobe, near the core, the shape of the spectrum shows a curvature in the highest frequency region, where $\alpha$ reaches values of $\sim 1.3-1.5$. Such steepening is in agreement with the synchrotron aging of the emitting relativistic electrons.

The brightness profiles of the lobes of these sources also have been obtained. For 3C 35 the brightness profile shows a constant trend at 74 and $327 \mathrm{MHz}$ while at $1.4 \mathrm{GHz}$, a decrease of intensity is evident from the hot spot to the inner of the lobe. The brightness profile of $3 \mathrm{C} 223$ is characterized by a decrease of brightness from the hot spot to the inner lobe at all the frequencies analyzed. As radiative losses at long wavelengths are negligible, the different behaviours of the brightness profiles in the two sources might suggest that adiabatic losses are more relevant in 3C 223 than in $3 \mathrm{C} 35$.

A more detailed analysis is in progress (Orrù, PhD Thesis; Orrù et al., in prep.).

\section{References}

Lara, L., Márquez, I., Cotton, W. D., Feretti, L., Giovannini, G., Marcaide, J. M., \& Venturi, T. 2001, $A \mathscr{E} A, 378,826$ 